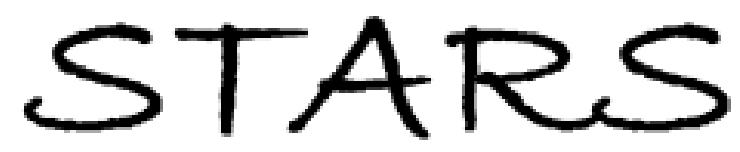

University of Central Florida

STARS

$1-1-1995$

\title{
Dark-Soliton Timing Jitter Caused By Fluctuations In Initial Pulse- Shape
}

\author{
N. -C. Panoiu \\ D. Mihalache \\ D. -M. Baboiu \\ University of Central Florida
}

Find similar works at: https://stars.library.ucf.edu/facultybib1990

University of Central Florida Libraries http://library.ucf.edu

This Article is brought to you for free and open access by the Faculty Bibliography at STARS. It has been accepted for inclusion in Faculty Bibliography 1990s by an authorized administrator of STARS. For more information, please contactSTARS@ucf.edu.

\section{Recommended Citation}

Panoiu, N. -C.; Mihalache, D.; and Baboiu, D. -M., "Dark-Soliton Timing Jitter Caused By Fluctuations In Initial Pulse-Shape" (1995). Faculty Bibliography 1990s. 1429.

https://stars.library.ucf.edu/facultybib1990/1429

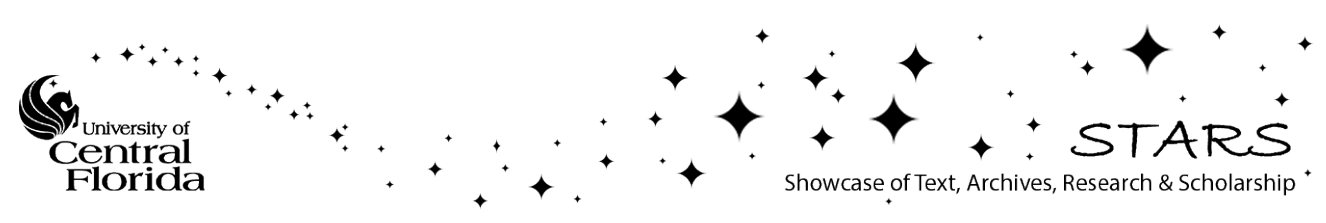




\title{
Dark-soliton timing jitter caused by fluctuations in initial pulse shape
}

\author{
N.-C. Panoiu, ${ }^{1,2}$ D. Mihalache, ${ }^{1,3}$ and D.-M. Baboiu ${ }^{4,1}$ \\ ${ }^{1}$ Department of Theoretical Physics, Institute of Atomic Physics, P.O. Box MG-6, Bucharest, Romania \\ ${ }^{2}$ Physics Department, New York University, 4 Washington Place, New York, New York 10003 \\ ${ }^{3}$ Department of Signal Theory and Communications, Polytechnic University of Catalonia, 08034 Barcelona, Spain \\ ${ }^{4}$ Center for Research in Electro-Optics and Lasers, University of Central Florida, Suite 400, 12424 Research Parkway, \\ Orlando, Florida 32826
}

(Received 29 March 1995)

\begin{abstract}
The dark-soliton timing jitters caused by fluctuations in either the soliton initial phase angle or the background amplitude when such a soliton propagates in a monomode optical fiber under the influence of the stimulated Raman scattering are investigated and compared with those that exist when the stimulated Raman scattering is not present. In addition, it is demonstrated that in the presence of the stimulated Raman scattering, there exists a distance at which, for the negative soliton initial phase angle, the dark-soliton timing jitter caused by fluctuations in the background amplitude becomes zero.
\end{abstract}

PACS number(s): 42.50.Rh

The possibility of propagation of dark solitons in the region of normal (positive) group-velocity dispersion (GVD) in monomode optical fibers has been theoretically predicted by Hasegawa and Tappert [1] and has been successfully demonstrated in a number of elegant experiments [2]. Since then, the properties of dark-soliton propagation in optical fibers have been thoroughly studied in several works [3-10] and have been reviewed in two recent articles $[11,12]$. The darksoliton propagation in real fibers is subject to various kinds of perturbations such as the third-order dispersion, the absorption loss, the stimulated Raman scattering (SRS), and the stochastic perturbations. The stochastic perturbations could be classified into two groups [13]: the homogeneous one, when the stochasticity originates in the random perturbations in the fiber itself, and the inhomogeneous one, when the stochasticity is present in the input pulse to the fiber. In the latter case, the propagation is entirely deterministic; only the initial parameters of the pulse are random quantities. One major source of the inhomogeneous stochasticity is the amplified spontaneous emission (ASE) noise. The ASE noise produces a soliton timing jitter (the Gordon-Haus effect) and the theory of this effect has been given in [14] for bright solitons and in [15] for dark ones.

In this paper we study the dark-soliton timing jitter caused by fluctuations in the soliton initial parameters when such a soliton propagates in a monomode optical fiber in the presence of the SRS. We focus on the influence of the SRS on dark-soliton timing jitter because, as it is well known, the SRS has the most deleterious effects for dark-soliton propagation in optical fibers.

For the normal (positive) GVD the propagation of picosecond optical pulses in monomode optical fibers is governed by the famous nonlinear Schrödinger equation

$$
i \frac{\partial \psi}{\partial z}-\frac{1}{2} \frac{\partial^{2} \psi}{\partial t^{2}}+|\psi|^{2} \psi=0
$$

where $z$ is the normalized distance along the fiber, $t$ is the normalized time, and $\psi$ is the normalized complex amplitude of the pulse envelope. The normalized units are well known: $z=\beta_{2} Z / T_{0}^{2}$ and $t=\left(T-Z / v_{g}\right) / T_{0}$, where $\beta_{2}$ is the GVD coefficient, $T_{0}$ is the pulse width $\left(T_{\mathrm{FWHM}}=1.763 T_{0}\right.$, where FWHM is the full width at half maximum), $v_{g}$ is the groupvelocity, and $Z$ and $T$ are the physical distance and time, respectively.

When we deal with subpicosecond optical pulses, an accurate treatment of their propagation calls for the introduction of new terms in Eq. (1). Thus, to take into account the SRS, we have to modify Eq. (1) as follows:

$$
i \frac{\partial \psi}{\partial z}-\frac{1}{2} \frac{\partial^{2} \psi}{\partial t^{2}}+|\psi|^{2} \psi=\sigma \psi \frac{\partial\left(|\psi|^{2}\right)}{\partial t},
$$

where $\sigma=T_{R} / T_{0}$ and $T_{R}$ is related to the slope of the Raman gain $\left(T_{R} \simeq 6 \mathrm{fs}\right)[16]$.

It is well known that Eq. (1) supports the modulationally stable continuous-wave (cw) background $\psi=\rho e^{i \rho^{2} z}, \rho$ being the background amplitude. Because the Raman term tends to zero when $t \rightarrow \pm \infty$, it does not affect the background amplitude $\rho$. Now, in order to simplify our further analysis, we make the transformation $\psi(z, t)=e^{i \rho^{2} z} u(z, t)$. Thus Eq. (2) becomes

$$
i \frac{\partial u}{\partial z}-\frac{1}{2} \frac{\partial^{2} u}{\partial t^{2}}+\left(|u|^{2}-\rho^{2}\right) u=\sigma u \frac{\partial\left(|u|^{2}\right)}{\partial t} .
$$

A dark soliton of Eq. (3) without the Raman term $(\sigma=0)$ is a kink-type soliton that connects two stable background waves with the same amplitude but with different phases and has the form

$$
u_{s}(z, t)=\rho(\cos \phi \tanh \zeta-i \sin \phi)
$$

where $\zeta=\eta(t-\Omega z), \quad \eta=\rho \cos \phi$, and $\Omega=\rho \sin \phi$. Such a soliton is characterized by its phase angle $\phi(|\phi|<\pi / 2)$, which describes the darkness of the soliton through the simple relation

$$
\left|u_{s}\right|^{2}=\rho^{2}\left(1-\cos ^{2} \phi \operatorname{sech}^{2} \zeta\right)
$$


This represents a dip with the depth equal to $\eta^{2}=\rho^{2} \cos ^{2} \phi$ and the velocity $\Omega=\rho \sin \phi$, which moves on a background intensity with the amplitude $\rho^{2}$. When $\phi \ll 1$ we call this soliton a dark-gray one and when $\phi= \pm \pi / 2 \mp \epsilon, \epsilon \ll 1$, we call it a light-gray one [8]. From Eq. (4) we see that the dark-gray soliton has a small velocity and large amplitude while the light-gray one has a large velocity and small amplitude so that it could be considered as a small excitation on the cw background.

For the dark soliton (4) the shift in the inverse group velocity is $\beta_{2} \Omega / T_{0}$, that is, $1 / V_{g}=1 / v_{g}+\beta_{2} \Omega / T_{0}$. Thus the soliton arrival time $T^{0}$ in the absence of the Raman term is given by the formula

$$
T^{0}=\int_{0}^{Z} \frac{d Z^{\prime}}{V_{g}}=\left(\frac{Z}{v_{g}}+\frac{\beta_{2} \rho \sin \phi_{0}}{T_{0}}\right) Z,
$$

where $\phi_{0}$ represents the soliton initial phase angle. Equation (6) can be put in its dimensionless form as

$$
t^{0}=\frac{Z}{v_{g} T_{0}}+\rho \sin \left(\phi_{0}\right) z .
$$

Now, let us suppose that either the soliton initial phase angle $\phi_{0}$ or the background amplitude $\rho$ exhibits fluctuations with standard deviations $\Delta \phi_{0}$ and $\Delta \rho$, respectively. As a consequence, the soliton arrival time $t^{0}$ exhibits fluctuations (the so-called soliton timing jitter) given by

$$
\Delta t_{\phi}^{0}=\rho \cos \left(\phi_{0}\right) z \Delta \phi_{0}, \quad \Delta t_{\rho}^{0}=\left|\sin \phi_{0}\right| z \Delta \rho .
$$

Next we calculate the soliton timing jitter due to fluctuations in either the soliton initial phase angle $\phi_{0}$ or the background amplitude $\rho$ in the presence of the SRS. For this purpose we have to establish the dependence of the phase angle $\phi$ on the normalized propagation distance $z$. In order to derive this dependence we use the Hamiltonian approach (see, e.g., Ref. [17] and the references therein). It is well known that Eq. (3), with $\sigma=0$ and finite boundary conditions, is a Hamiltonian dynamical system. This means that it is equivalent to the Hamilton equations

$$
\frac{\partial u}{\partial z}=\{H, u\}, \quad \frac{\partial u^{*}}{\partial z}=\left\{H, u^{*}\right\}
$$

where the Poisson brackets are defined as

$$
\{F, G\}=i \int_{-\infty}^{\infty}\left(\frac{\delta F}{\delta u} \frac{\delta G}{\delta u^{*}}-\frac{\delta F}{\delta u^{*}} \frac{\delta G}{\delta u}\right) d t,
$$

where $F$ and $G$ are functionals of $u$ and $u^{*}$ and the Hamiltonian $H$ is taken as

$$
H=\frac{1}{2} \int_{-\infty}^{\infty}\left[\frac{\partial u}{\partial t} \frac{\partial u^{*}}{\partial t}+\left(|u|^{2}-\rho^{2}\right)^{2}\right] d t .
$$

In these formulas the asterisk stands for the complex conjugate.

It is known that in the case of dark solitons the presence of certain perturbations could lead to the creation of new solitons with small amplitudes and large velocities. These new solitons correspond to zeros of the scattering matrix situated at the edges of the allowed discrete spectrum of the inverse problem. In the presence of the Raman term Eq. (3) ceases to be a Hamiltonian system, so that the phase angle $\phi$ is no longer a constant parameter becoming a $z$-dependent function. We suppose that the Raman term is a small perturbation so that we can apply the adiabatic approximation. In this approximation the emission of radiation and the creation of new solitons are disregarded. Thus we start from the Hamiltonian of the unperturbed system $(\sigma=0)$, which for the dark-soliton solution (4) takes the value

$$
H_{s}=\frac{4 \rho^{3}}{3} \cos ^{3} \phi
$$

Next, by using Eqs. (3) and (11), we calculate the derivative of $H$ with respect to $z$,

$$
\frac{d H}{d z}=i \frac{\sigma}{2} \int_{-\infty}^{\infty} \frac{\partial^{2}\left(|u|^{2}\right)}{\partial t^{2}}\left(u * \frac{\partial u}{\partial t}-u \frac{\partial u^{*}}{\partial t}\right) d t .
$$

For the dark-soliton solution this equation gives

$$
\frac{d H_{s}}{d z}=-\frac{16}{15} \sigma \rho^{6} \cos ^{5} \phi \sin \phi,
$$

so that the resulting equation describing the evolution of the soliton phase angle $\phi$ with respect to $z$ can be written as $[8,9]$

$$
\frac{d \phi}{d z}=\frac{4}{15} \sigma \rho^{3} \cos ^{3} \phi .
$$

This expression shows us that the soliton phase angle $\phi$ can only increase with distance under the Raman perturbation, so if the initial phase angle $\phi_{0}$ is positive (soliton with positive velocity), the soliton depth $\eta$ decreases, leading to the soliton decay, and if the initial phase angle $\phi_{0}$ is negative (soliton with negative velocity), the soliton, in a first stage, will turn into a black one (soliton with zero velocity) and then its phase angle continues to grow, leading also to soliton decay. In other words, Eq. (15) shows that the SRS leads to a soliton self-frequency shift and soliton decay. These effects have been observed experimentally in [3] and explained analytically in [18] in the small-amplitude limit.

By integrating Eq. (15) with respect to $z$ we obtain the phase angle $\phi=\phi\left(z, \phi_{0}\right)$ as the solution of the implicit equation

$$
\begin{aligned}
F\left(\phi, z, \phi_{0}, \rho\right)= & \frac{\sin \phi}{\cos ^{2} \phi}+\ln \frac{1+\sin \phi}{\cos \phi}-\frac{\sin \phi_{0}}{\cos ^{2} \phi_{0}} \\
& -\ln \frac{1+\sin \phi_{0}}{\cos \phi_{0}}-\frac{8}{15} \sigma \rho^{3} z=0,
\end{aligned}
$$

where $\phi_{0}=\phi(0)$ is the soliton initial phase angle. The arrival time $T^{\sigma}$ in the presence of the perturbation is

$$
T^{\sigma}=\int_{0}^{Z} \frac{d Z^{\prime}}{V_{g}}=\frac{Z}{v_{g}}+\frac{\beta_{2} \rho}{T_{0}} \int_{0}^{Z} \sin \phi d Z^{\prime}
$$

or in its dimensionless form 


$$
t^{\sigma}=\frac{Z}{v_{g} T_{0}}+\rho \int_{0}^{z} \sin \phi\left(z^{\prime}\right) d z^{\prime}
$$

When either the soliton initial phase angle $\phi_{0}$ or the background amplitude $\rho$ fluctuates, it is obvious from Eq. (18) that the soliton arrival time $t^{\sigma}$ fluctuates, that is, a soliton timing jitter arises.

In the case of soliton timing jitter caused by fluctuations in the soliton initial phase angle $\phi_{0}$, we have, from Eq. (18)

$$
\Delta t_{\phi}^{\sigma}=\rho\left|\int_{0}^{z} \cos (\phi) \frac{\partial \phi}{\partial \phi_{0}} d z^{\prime}\right| \Delta \phi_{0} .
$$

Taking into account that from Eq. (16) we have

$$
\frac{\partial \phi}{\partial \phi_{0}}=\frac{\cos ^{3} \phi}{\cos ^{3} \phi_{0}}
$$

we obtain

$$
\begin{aligned}
\Delta t_{\phi}^{\sigma} & =\left(\frac{\rho}{\cos ^{3} \phi_{0}} \int_{0}^{z} \cos ^{4} \phi\left(z^{\prime}\right) d z^{\prime}\right) \Delta \phi_{0} \\
& =\frac{15}{4 \sigma \rho^{2} \cos ^{3} \phi_{0}}\left[\sin \phi(z)-\sin \phi_{0}\right] \Delta \phi_{0} .
\end{aligned}
$$

We note that in deriving this result we made use of Eq. (15). In order to compare the timing jitter $\Delta t_{\phi}^{\sigma}$ in the presence of the SRS with the timing jitter $\Delta t_{\phi}^{0}$ in its absence we consider the difference between them

$$
\Delta t_{\phi}^{\sigma}-\Delta t_{\phi}^{0}=\left[\frac{\rho}{\cos ^{3} \phi_{0}} \int_{0}^{z}\left[\cos ^{4} \phi\left(z^{\prime}\right)-\cos ^{4} \phi_{0}\right] d z^{\prime}\right] \Delta \phi_{0} .
$$

Bearing in mind that the phase angle $\phi$ is an increasing function of $z$, we deduce from Eq. (22) that, for positive values of $\phi_{0}$, the timing jitter $\Delta t_{\phi}^{\sigma}$ is less than the timing jitter $\Delta t_{\phi}^{0}$, while for negative values of $\phi_{0}$ there is a threshold value of the propagation distance $z_{\text {th }}^{\phi}$ so that for distances under this threshold $\Delta t_{\phi}^{\sigma}>\Delta t_{\phi}^{0}$ and for distances above this threshold $\Delta t_{\phi}^{\sigma}<\Delta t_{\phi}^{0}$. This is illustrated in Figs. 1(a) and 1(b). In Figs. 1(a) and 1(b) the soliton initial phase angle is $\phi_{0}=\pi / 6$ and $\phi_{0}=-\pi / 9$, respectively. The other parameters are $\rho=1$ and $\sigma=0$ (dashed lines) or $\sigma=0.1$ (solid lines). From Fig. 1(b) we evaluate $z_{\text {th }}^{\phi} \simeq 45$.

In the case of soliton timing jitter caused by fluctuations in the background amplitude $\rho$, from Eq. (18) we have

$$
\Delta t_{\rho}^{\sigma}=\left|\int_{0}^{z} \sin \phi\left(z^{\prime}\right) d z^{\prime}+\rho \int_{0}^{z} \cos (\phi) \frac{\partial \phi}{\partial \rho} d z^{\prime}\right| \Delta \rho .
$$

Taking into account that from Eq. (16) we have

$$
\frac{\partial \phi}{\partial \rho}=\frac{4}{5} \sigma \rho^{2} z \cos ^{3} \phi
$$

after simple calculations Eq. (23) becomes

$$
\Delta t_{\rho}^{\sigma}=\left|3 z \sin \phi(z)-2 \int_{0}^{z} \sin \phi\left(z^{\prime}\right) d z^{\prime}\right| \Delta \rho .
$$

(a)

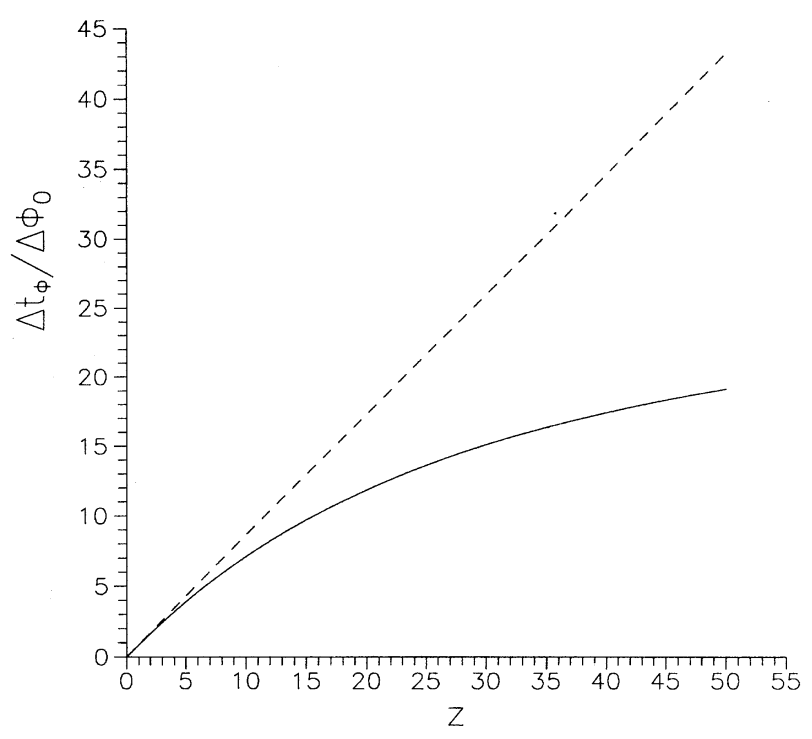

(b)

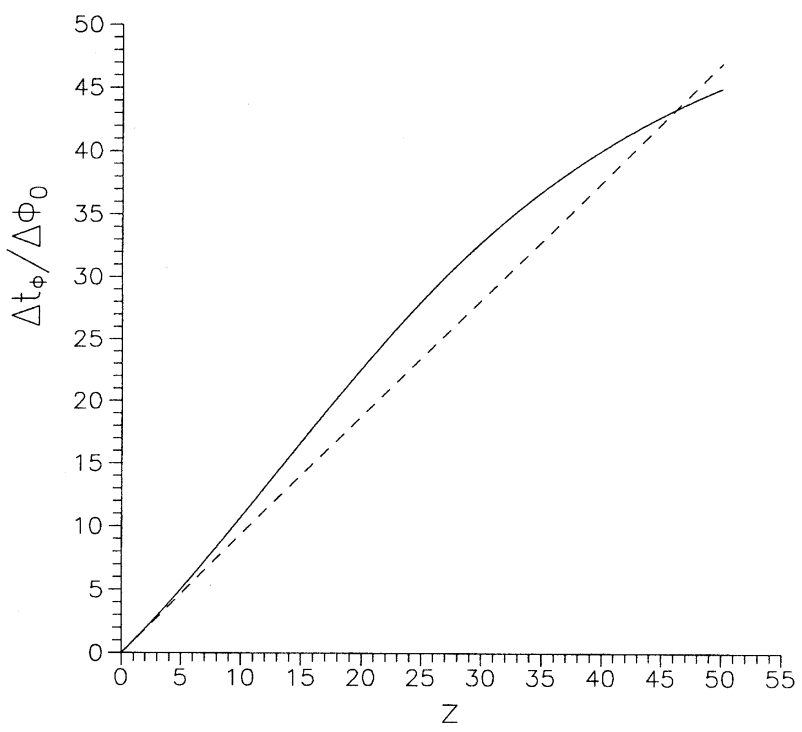

FIG. 1. Normalized soliton timing jitter caused by fluctuations in the soliton initial phase angle. Here $\rho=1, \sigma=0$ (dashed lines), and $\sigma=0.1$ (solid lines). (a) $\phi_{0}=\pi / 6$ and (b) $\phi_{0}=-\pi / 9$.

Now we compare the soliton timing jitter with and without SRS. The analysis depends on the sign of the soliton initial phase angle $\phi_{0}$.

In the case $\phi_{0}>0$ we have

$$
\Delta t_{\rho}^{0}=\sin \left(\phi_{0}\right) z \Delta \rho
$$

and

$$
\Delta t_{\rho}^{\sigma}=\left(3 z \sin \phi(z)-2 \int_{0}^{z} \sin \phi\left(z^{\prime}\right) d z^{\prime}\right) \Delta \rho,
$$

so that 
(a)

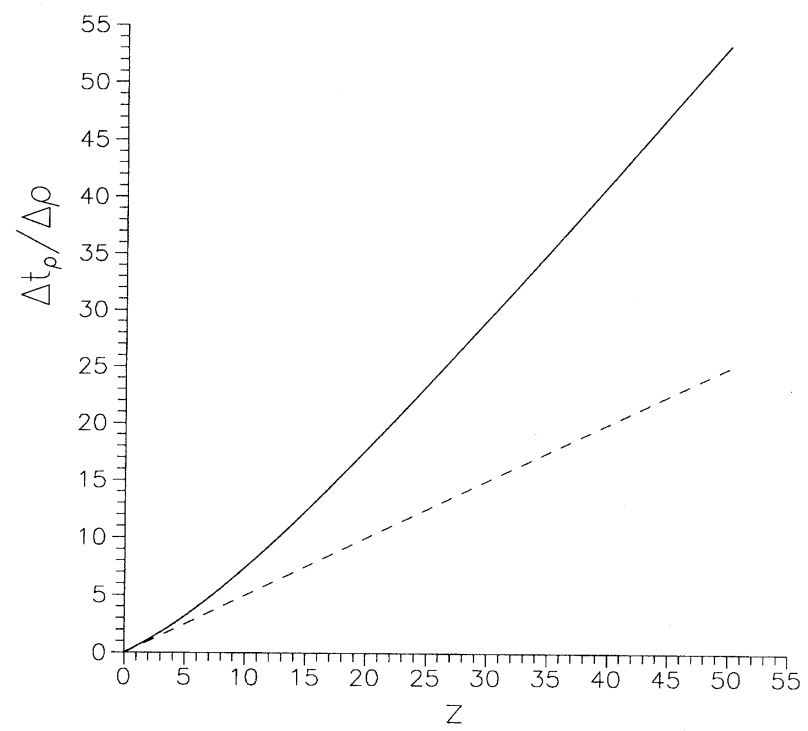

(b)

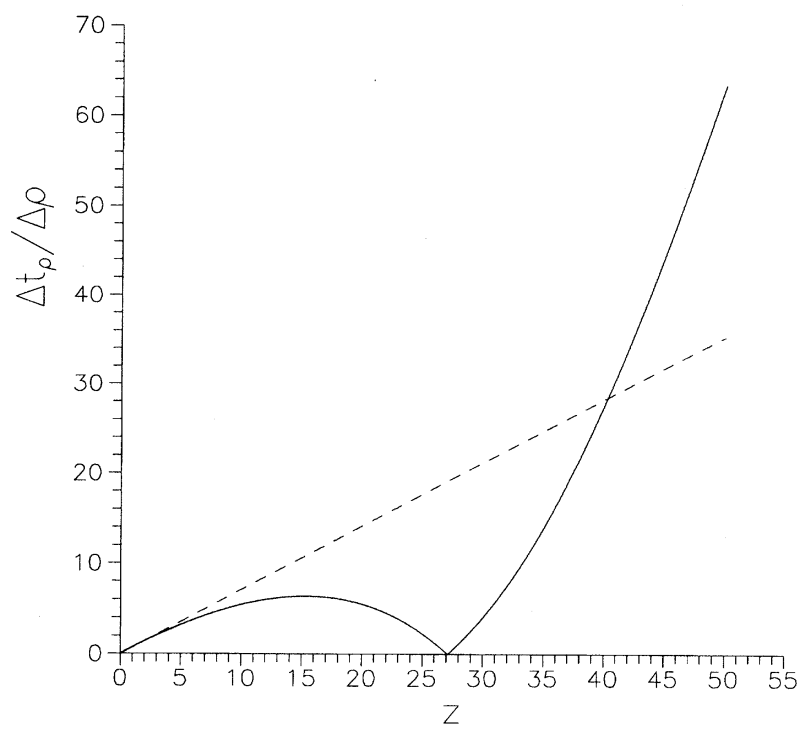

FIG. 2. Normalized soliton timing jitter caused by fluctuations in the background amplitude. Here $\rho=1, \sigma=0$ (dashed lines), and $\sigma=0.1$ (solid lines). (a) $\phi_{0}=\pi / 6$ and (b) $\phi_{0}=-\pi / 4$.

$$
\begin{aligned}
\Delta t_{\rho}^{\sigma}-\Delta t_{\rho}^{0}= & {\left[z\left[\sin \phi(z)-\sin \phi_{0}\right]\right.} \\
& \left.+2\left(z \sin \phi(z)-\int_{0}^{z} \sin \phi\left(z^{\prime}\right) d z^{\prime}\right)\right] \Delta \rho .
\end{aligned}
$$

This formula and the fact that $\phi$ is an increasing function of $z$ show us that in this case $\Delta t_{\rho}^{\sigma}>\Delta t_{\rho}^{0}$. This is illustrated in Fig. 2(a).
In the case $\phi_{0}<0$ we consider the function $f(z)$ defined as

$$
f(z)=3 z \sin \phi(z)-2 \int_{0}^{z} \sin \phi\left(z^{\prime}\right) d z^{\prime} .
$$

The derivative of this function with respect to $z$ is $f^{\prime}(z)=\sin \phi(z)+3 z \cos \phi(z) \phi^{\prime}(z)$, where the prime means differentiation with respect to $z$. From this expression it can be seen that $f^{\prime}(0)<0$, so that, by taking into account that $f(0)=0$, we conclude that for small values of $z$ the function $f(z)$ is negative. In turn, for large values of $z$ the function $f(z)$ becomes positive. So there exists a value $z_{0}$ at which $f\left(z_{0}\right)=0$, that is, $\Delta t_{\rho}^{\sigma}\left(z_{0}\right)=0$.

Now let us compare the jitters $\Delta t_{\rho}^{\sigma}$ and $\Delta t_{\rho}^{0}$. For $0<z<z_{0}$ we have

$$
\begin{aligned}
\Delta t_{\rho}^{\sigma}-\Delta t_{\rho}^{0}= & {\left[2\left(\int_{0}^{z} \sin \phi\left(z^{\prime}\right) d z^{\prime}-z \sin \phi(z)\right)\right.} \\
& \left.+z\left(\sin \phi_{0}-\sin \phi\right)\right] \Delta \rho,
\end{aligned}
$$

so that $\Delta t_{\rho}^{\sigma}<\Delta t_{\rho}^{0}$. For $z>z_{0}$ we have

$$
\Delta t_{\rho}^{\sigma}-\Delta t_{\rho}^{0}=\left[3 z \sin \phi(z)-2 \int_{0}^{z} \sin \phi\left(z^{\prime}\right) d z^{\prime}+z \sin \phi_{0}\right] \Delta \rho .
$$

From this expression it can be inferred that there exists a threshold propagation distance $z_{\mathrm{th}}^{\rho}>z_{0}$ so that $\Delta t_{\rho}^{\sigma}<\Delta t_{\rho}^{0}$ when $z<z_{\text {th }}^{\rho}$ and $\Delta t_{\rho}^{\sigma}>\Delta t_{\rho}^{0}$ when $z>z_{\text {th }}^{\rho}$. This behavior is illustrated in Fig. 2(b). From this figure we evaluate $z_{0} \simeq 27$ and $z_{\mathrm{th}}^{\rho} \simeq 40$.

Finally, we briefly discuss the soliton timing jitters $\Delta t_{\phi}^{\sigma}, \Delta t_{\phi}^{0}, \Delta t_{\rho}^{\sigma}, \Delta t_{\rho}^{0}$ in the limit cases of dark-gray and lightgray solitons.

In the case of dark-gray solitons $\left(\phi_{0} \ll 1\right)$ the formulas (8) become

$$
\Delta t_{\phi}^{0}=\rho\left(1-\frac{\phi_{0}^{2}}{2}\right) z \Delta \phi_{0}, \quad \Delta t_{\rho}^{0}=\left|\phi_{0}\right| z \Delta \rho
$$

In order to find the expressions for soliton timing jitters in the presence of SRS we first solve Eq. (15) in the limit $\phi, \phi_{0} \ll 1$. After simple calculations we obtain

$$
\phi=\frac{\phi_{0}+\sqrt{\frac{2}{3}} \tanh \left(\sqrt{\frac{3}{2}} \frac{4}{15} \sigma \rho^{3} z\right)}{1+\sqrt{\frac{3}{2}} \phi_{0} \tanh \left(\sqrt{\frac{3}{2}} \frac{4}{15} \sigma \rho^{3} z\right)} .
$$

By introducing this expression in Eqs. (21) and (25) we obtain

$$
\Delta t_{\phi}^{\sigma}=\Delta t_{\phi}^{0}-\frac{8}{15} \sigma \phi_{0} \rho^{4} z^{2} \Delta \phi_{0}+O\left(\sigma^{2}\right)
$$

and 


$$
\Delta t_{\rho}^{\sigma}=\Delta t_{\rho}^{0}+\operatorname{sgn}\left(\phi_{0}\right) \frac{8}{15} \sigma \rho^{3} z^{2} \Delta \rho+O\left(\sigma^{2}\right)
$$

where $\operatorname{sgn}\left(\phi_{0}\right)=1$ for $\phi_{0}>0$ and $\operatorname{sgn}\left(\phi_{0}\right)=-1$ for $\phi_{0}<0$.

We study the light-gray soliton case $\left(\phi_{0}= \pm \pi / 2\right.$ $\left.\mp \epsilon_{0}, \epsilon_{0} \ll 1\right)$ with positive velocity $\left(\phi_{0}=\pi / 2-\epsilon_{0}\right.$, $\left.\epsilon_{0} \ll 1\right)$. The analysis of the other case is similar. From the formulas (8) we deduce

$$
\Delta t_{\phi}^{0}=\rho \epsilon_{0} z \Delta \phi_{0}, \quad \Delta t_{\rho}^{0}=\left(1-\frac{\epsilon_{0}^{2}}{2}\right) z \Delta \rho .
$$

As before, we derive the expression for the small parameter $\epsilon$, which corresponds to the soliton phase angle $\phi$,

$$
\epsilon=\epsilon_{0}\left(1+\frac{8}{15} \sigma \rho^{3} \epsilon_{0}^{2} z\right)^{-1 / 2}
$$

and for the timing jitters in the presence of the SRS

$$
\begin{gathered}
\Delta t_{\phi}^{\sigma}=\Delta t_{\phi}^{0}-\frac{8}{15} \sigma \rho^{4} \epsilon_{0}^{3} z^{2} \Delta \phi_{0}+O\left(\sigma^{2}\right), \\
\Delta t_{\rho}^{\sigma}=\Delta t_{\rho}^{0}+\frac{8}{15} \sigma \rho^{3} \epsilon_{0}^{4} z^{2} \Delta \rho+O\left(\sigma^{2}\right) .
\end{gathered}
$$

In conclusion, we have studied the dark-soliton timing jitter caused by fluctuations in the soliton initial pulse shape. In our analysis we first supposed that the dark-solitons propagate unperturbed and we have calculated the soliton timing jitters caused by fluctuations in either the soliton initial phase angle $\phi_{0}$, that is, $\Delta t_{\phi}^{0}$, or the background amplitude $\rho$, that is, $\Delta t_{\rho}^{0}$. Then we have calculated the same jitters, supposing that the dark solitons propagate under the influence of the SRS, that is, $\Delta t_{\phi}^{\sigma}$ and $\Delta t_{\rho}^{\sigma}$. It has been established that for dark solitons with a positive soliton initial phase angle $\left(\phi_{0}>0\right)$ the soliton timing jitter caused by fluctuations in soliton initial phase angle is smaller in the presence of SRS than in the unperturbed case, while the soliton timing jitter caused by fluctuations in background amplitude is larger in the presence of SRS than in the unperturbed case. Furthermore, for solitons with $\phi_{0}>0$ both jitters are always strictly positive. For dark solitons with a negative soliton initial phase angle $\left(\phi_{0}<0\right)$, it has been established that there exists a threshold propagation distance $z_{\text {th }}^{\phi}$ so that $\Delta t_{\phi}^{\sigma}>\Delta t_{\phi}^{0}$ for $0<z<z_{\text {th }}^{\phi}$ and $\Delta t_{\phi}^{\sigma}<\Delta t_{\phi}^{0}$ for $z>z_{\text {th }}^{\phi}$ and a threshold propagation distance $z_{\text {th }}^{\rho}$ so that $\Delta t_{\rho}^{\sigma}<\Delta t_{\rho}^{0}$ for $0<z<z_{\text {th }}^{\rho}$ and $\Delta t_{\rho}^{\sigma}>\Delta t_{\rho}^{0}$ for $z>z_{\text {th }}^{\rho}$. In addition, it has been demonstrated that, for solitons with $\phi_{0}<0$, there exists a propagation distance $z_{0}$ at which $\Delta t_{\rho}^{\sigma}$ becomes zero while $\Delta t_{\phi}^{\sigma}$ is strictly positive. Finally, we have calculated the soliton timing jitters in the two limit cases: dark-gray and lightgray solitons.

One of the authors (D.M.) acknowledges the partial support of the European Community through the Human Capital and Mobility Program and the hospitality of the Supercomputer Center of Catalonia. D.M. is also grateful to Professor L. Torner for helpful discussions.
[1] A. Hasegawa and F. Tappert, Appl. Phys. Lett. 23, 171 (1973).

[2] Ph. Emplit, J.P. Hamaide, F. Reynaud, G. Froehly, and A. Barthelemy, Opt. Commun. 62, 374 (1987); D. Krökel, N.J. Halas, G. Giuliani, and D. Grischkowski, Phys. Rev. Lett. 60, 29 (1988); A.M. Weiner, J.P. Heritage, R.J. Hawkins, R.N. Thurston, E.M. Kirschner, D.E. Leaird, and W.J. Tomlinson, ibid. 61, 2445 (1988).

[3] A.M. Weiner, R.N. Thurston, W.J. Tomlinson, J.P. Heritage, D.E. Leaird, E.M. Kirschner, and R.J. Hawkins, Opt. Lett. 14, 868 (1989).

[4] K.J. Blow and N.J. Doran, Phys. Lett. A 107, 55 (1985).

[5] W.J. Tomlinson, R.J. Hawkins, A.M. Weiner, J.P. Heritage, and R.N. Thurston, J. Opt. Soc. Am. B 6, 329. (1989).

[6] S.A. Gredeskul and Yu.S. Kivshar, Phys. Rev. Lett. 62, 977 (1989); Opt. Lett. 14, 1281 (1989).

[7] J.E. Rothenberg, Opt. Commun. 82, 107 (1991); J.E. Rothenberg and H.K. Heinrich, Opt. Lett. 17, 261 (1992).

[8] I.M. Uzunov and V.S. Gerdjikov, Phys. Rev. A 47, 1582 (1993).
[9] Yu.S. Kivshar and X. Yang, Phys. Rev. E 49, 1657 (1994).

[10] D. Mihalache and N.-C. Panoiu, J. Phys. A 26, 2679 (1993); D. Mihalache, F. Lederer, and D.-M. Baboiu, Phys. Rev. A 47, 3285 (1993); N. Akhmediev and A. Ankiewicz, ibid. 47, 3213 (1993); L. Gagnon, J. Opt. Soc. Am. B 10, 469 (1993).

[11] A.M. Weiner, in Optical Solitons: Theory and Experiment, edited by J.R. Taylor (Cambridge University Press, Cambridge, 1992).

[12] Yu.S. Kivshar, IEEE J. Quantum Electron. 28, 250 (1993).

[13] J.N. Elgin, Phys. Lett. A 110, 441 (1985); Phys. Rev. A 47, 4331 (1993); Opt. Lett. 18, 10 (1993).

[14] J.P. Gordon and H.A. Haus, Opt. Lett. 11, 665 (1986).

[15] Yu.S. Kivshar, M. Haelterman, Ph. Emplit, and J.P. Hamaide, Opt. Lett. 19, 19 (1994).

[16] H.A. Haus and M. Nakazawa, J. Opt. Soc. Am. B 4, 652 (1987).

[17] Yu.S. Kivshar and B.A. Malomed, Rev. Mod. Phys. 61, 763 (1989).

[18] Yu.S. Kivshar and V.V. Afanasjev, Opt. Lett. 16, 285 (1991). 